\title{
TEMPERATURE SENSITIVE PAINT APPLICATION FOR INVESTIGATION OF BOUNDARY LAYER TRANSITION IN SHORT-DURATION WIND TUNNELS
}

\author{
V. Borovoy, V. Mosharov, A. Noev, and V. Radchenko \\ Central Aerohydrodynamic Institute (TsAGI) \\ Zhukovsky Str. 1, Zhukovsky, Moscow region 140180, Russia
}

\begin{abstract}
Application of Temperature Senstive Paint (TSP) technology for investigation of boundary layer transition in short-duration wind tunnels is presented. Investigations were made on sharp and blunted flat plates in UT-1 wind tunnel of TsAGI operated in Ludwieg scheme at Mach numbers 5,6 , and 8 (Reynolds numbers from $5.5 \cdot 10^{6}$ to $26 \cdot 10^{6}$ ). Both natural and induced boundary layer transitions were investigated.
\end{abstract}

\section{BACKGROUND}

Temperature Sensitive Paint method is a very effective optical technique for heat flux measurements. It provides the pattern of heat transfer rates on the whole visible model surface that is essential for three-dimensional (3D) flows. In few recent years, this technique is successfully developed in TsAGI for short-duration wind tunnels $[1,2]$.

Temperature Sensitive Paint is a specially developed paint containing specific luminophore which luminescence depends on temperature. Being excited by the light of appropriate spectral range, the TSP emits the luminescent light of longer wavelength range, and luminescent light intensity decreases with an increase of temperature. Thus, the temperature distribution can be determined in each point of investigated surface from noncontact optical measurement of TSP luminescence intensity by acquiring an image of the whole investigated surface. But luminescence intensity depends not only on temperature but also on excitation light intensity and TSP thickness. To exclude an effect of excitation light intensity and TSP thickness, it is necessary to acquire second reference image of investigated surface at known temperature. Normalization of active image (acquired at unknown temperature distribution) on reference image exclude an effects of

This is an Open Access article distributed under the terms of the Creative Commons Attribution-Noncommercial License 3.0, which permits unrestricted use, distribution, and reproduction in any noncommercial medium, provided the original work is properly cited. 
excitation light and TSP thickness distributions. Unfortunately excitation light distribution may be changed because of model displacement and deformation and/or because of excitation light source instability. This effect can be excluded by usage of two-component (binary) TSP. Second reference component of such TSP is excited by the same excitation light and emits luminescent light in the spectral range different from spectral range of active component luminescence, and intensity of this light is insensitive to the temperature and depends only on excitation light intensity. Normalization of active component images on reference component images allows excluding any effect of excitation light intensity variation. Resultant image obtained from images of active and reference components acquired at known ('wind-off') and investigated ('wind-on') conditions depends only on temperature distribution on investigated surface and allows calculating this temperature distribution using TSP calibration characteristic.

To determine heat flux distribution, it is necessary to acquire temperature distributions at two time moments and to calculate heat transfer rate in each point of investigated surface using thermoconductivity properties of model material. In most cases, the global heat flux image can be calculated from temperature field measured by TSP using exact solution of one-dimensional (1D) heat transfer equation:

$$
\vartheta=1-\exp \left(\beta^{2}\right) \operatorname{ercf}(\beta) ; \quad \vartheta=\frac{T_{m}-T_{\text {in }}}{T_{r}-T_{\text {in }}}
$$

where $T_{\text {in }}$ is the initial model temperature before wind tunnel start measured with thermocouple; $T_{m}$ is the model surface temperature at the moment $t$ after flow initialization measured by TSP; $T_{r}$ is the recovery temperature and is assumed $T_{r} \approx T_{0} ; \beta=h \sqrt{t} / \sqrt{\lambda c \rho}$ is the nondimensional heat flux ( $h$ is the heat transfer coefficient and $\sqrt{\lambda c \rho}$ is the thermal product of model material); and erfc is a complementary error function. Stanton number fields can be calculated as $\mathrm{St}=h /\left(\rho_{\infty} V_{\infty} C_{p}\right)$.

To minimize an influence of TSP on heat flux measurements, it is necessary to minimize TSP thickness that provides the minimal heat capacity of TSP layer, on one hand, and to minimize temperature distribution inside TSP layer, on the other, that provides the most accurate measurement of the temperature of upper surface of investigated model.

Heat transfer distribution is caused by various aerodynamic effects, such as shock waves, separations, boundary layer state, etc. This paper presents application of TSP technique for boundary layer transition investigations. Boundary layer transition can be detected with TSP technique because the heat flux in turbulent boundary layer is much higher than in laminar one.

Specific feature of short-duration wind tunnels is short period of stable flow parameters; hence, all measurements should be made within this short period. On one side, it means that model surface temperature will not reach too high 
values even at quite high stagnation temperature value that allows to use organicbased TSP. On the other side, the measurement system should be quite fast to provide the measurements in this short operation period.

\section{EXPERIMENTAL FACILITY AND THE MODEL}

Presented experiments were performed in UT-1 wind tunnel of TsAGI operated as Ludwieg tube. UT-1 wind tunnel is equipped with supersonic contoured nozzles for Mach numbers 5, 6, and 8. Total pressure was in the range of 7090 bar, and total temperature 510, 590, and $740 \mathrm{~K}$ depending on Mach number. Flow duration was $40 \mathrm{~ms}$.

Investigations of boundary layer transition were made on the flat plate 1 (Fig. 1) of 318.8-millimeter length and 150-millimeter width. Model was equipped with the sets of removable leading edges 3 . Ten removable leading edges of the plate had blunting from sharp to 5-millimeter radius, but only leading edges of 0-, 0.75- and 2-millimeter radii $(r)$ were used in present experiments. The model (flat plate) was made of steel while the investigated surface was covered by prepreg 2 to provide low thermoconductivity. In order to provide model strength, some part of the surface was not covered by prepreg, and small metal strips were remaining along the model borders. Sharp fences 4 were installed on both sides of the plate to prevent gas overflow from the lower plate surface.
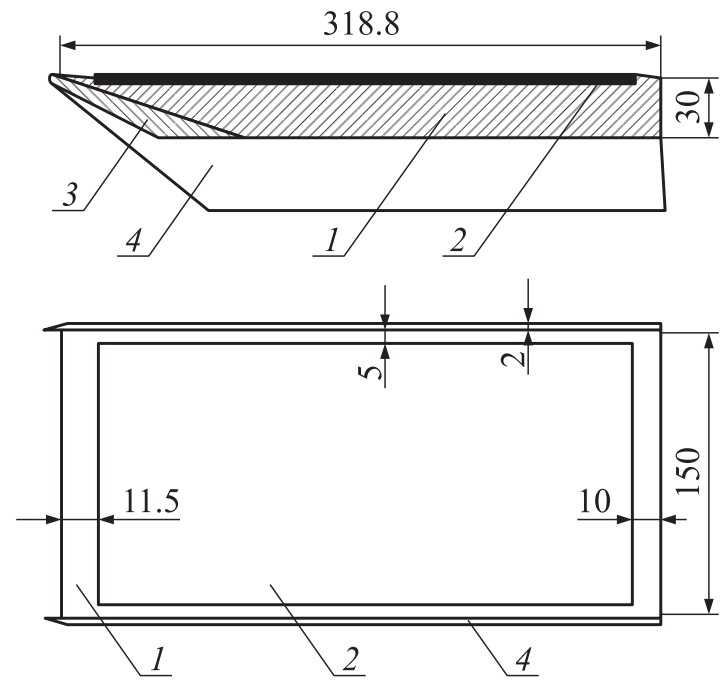

Figure 1 Model configuration. Dimensions are in millimeters 
Both natural and induced boundary layer transitions were investigated. Trips were made as small disks of 1-millimeter diameter and 0.2-millimeter thick that were placed in 3 rows on the distance from 15 to $25 \mathrm{~mm}$ from plate leading edge with the step of $3 \mathrm{~mm}$. Trips height was calculated by A. Skuratov based on $[3,4]$. Transition delay relative to generators position should be about $50 \mathrm{~mm}$ for $\mathrm{M}=6$ according to this calculation.

Plate surface was covered with specially chosen white basecoat first. Basecoat was chousing to have thermoinsulating properties as close to that of the model material as possible to exclude an influence of the basecoat on heat flux measurements. After basecoat drying, the trips were placed on the model surface. After that, the TSP was applied on the model surfaces by sprayer like an ordinary paint. Final thickness of dry TSP layer was about 3-5 $\mu \mathrm{m}$.

Experiments were made with trips first, then the generators were carefully removed from the model surface with minimal damaging of TSP layer and the same tests were repeated without any repairing of TSP layer. As a result, the final images without trips also have the marks of these generators.

\section{MEASUREMENT SYSTEM}

One of the problems of the present experiments is connected with large model displacement during a run due to aerodynamic load. This displacement leads to significant change of excitation light intensity on the model surface between 'windoff' and 'wind-on' images. To correct these changes, the binary (two-component) TSP was used. It contains two luminophors: first (active) luminophor is temperature sensitive (its luminescence decreases at the temperature increase with the rate of $3-5 \% /{ }^{\circ} \mathrm{C}$ ); the second one is temperature insensitive and is used as a reference for pixel-by-pixel correction of excitation light intensity changes. Both luminophors are excited simultaneously from one light source (ultraviolet (UV) flash lamps), but emits light of different spectral ranges that allows to separate luminescence of active and reference components easily by color glass filters. Two flash lamps connected serially to one power supply were used to provide more uniform illumination of the model. Flash lamps were equipped with UV glass filters installed in front of the lamps to pass only UV light and to cut off visible and infrared light from the lamp. Electrical power of flash lamps power supply could be varied up to $400 \mathrm{~J}$. Flash duration was less than $1 \mathrm{~ms}$. Light pulse was initiated with predetermined delay of $40 \mathrm{~ms}$ after flow initialization. For this purpose, the pulse signal from pressure sensor installed in the nozzle inlet was delayed by generator for required period.

Two CCD (charge coupled device) cameras of $1360 \times 1024$ pixel resolution were used to acquire temperature sensitive and reference images separately. Separation of sensitive and reference luminescence was provided by appropriate glass filters installed in front of camera lenses: red for sensitive component and blue for 
reference component. Cameras had electronic shutter and were synchronized with flash lamp that permited to acquire images at some level of ambient light. Both images were acquired simultaneously twice a run: before wind tunnel run at known temperature ('wind-off' images) and with predetermined delay of $40 \mathrm{~ms}$ after flow initialization ('wind-on' images). The ratio of these four images was converted to temperature field using appropriate calibration. Calibration of TSP was fulfilled in calibration setup on TSP sample prepared

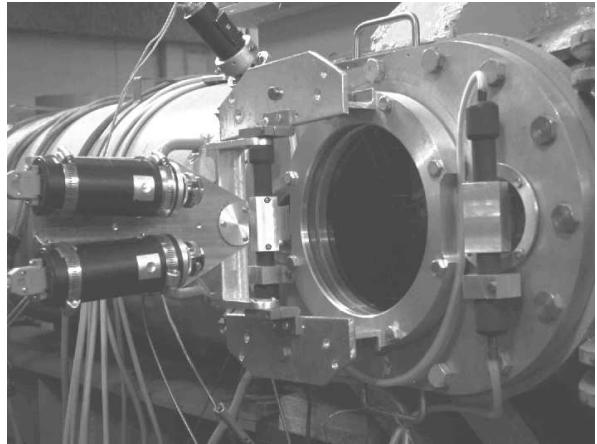

Figure 2 Flash lamps and CCD cameras mounted on the window of test section of UT-1 wind tunnel. (Refer color plate, p. I.) simultaneously with the model covering. Global heat transfer image was computed from temperature field assuming 1D heat transfer equation. Final results are presented as Stanton numbers fields.

Flash lamps and CCD cameras were mounted on the windows of test section as shown in Fig. 2. Distance from the lamps to the model was about $0.3 \mathrm{~m}$ and from CCD cameras - about $0.5 \mathrm{~m}$.

\section{RESULTS}

Stanton number distribution allows analyzing the state of boundary layer on the whole plate surface. A set of narrow strips of heat transfer growth are clearly visible near sharp leading edge at trip absence (Fig. $3 b$ ). These strips are transformed to the wedge-shaped of $9^{\circ}-10^{\circ}$ at some distance. Such heat transfer wedges are generated at any leading edge bluntness and all Mach numbers. These wedges are more intense in the central part of the plate, i. e., near flow center, that can be caused by flow disturbances that are concentrated on the axis of axisymmetric hypersonic nozzle.

Figure 4 presents the comparison of measured Stanton number sections with computed values for $\mathrm{M}=6$. In the lateral section located near the leading edge $(X=50 \mathrm{~mm})$ for the plate without trip, measured values are between the values computed for laminar and turbulent boundary layers on the whole plate surface except model boundaries where plate surface was not covered by thermoinsulating prepreg. This distribution shows high instability of St value, caused by instability of boundary layer transition. In the later sections, boundary layer is transformed to the turbulent state causing stabilization of Stanton number near turbulent values. In the axial section $(Z=0 \mathrm{~mm})$, the boundary layer transi- 


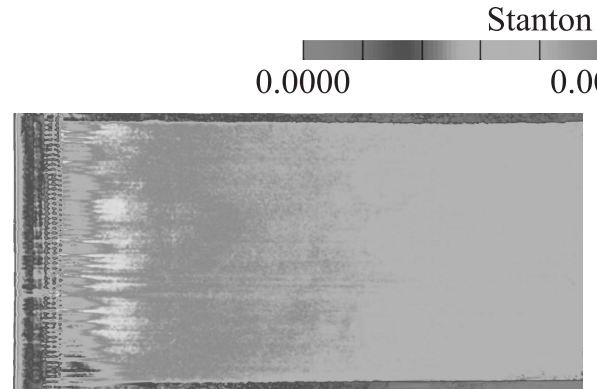

(a)

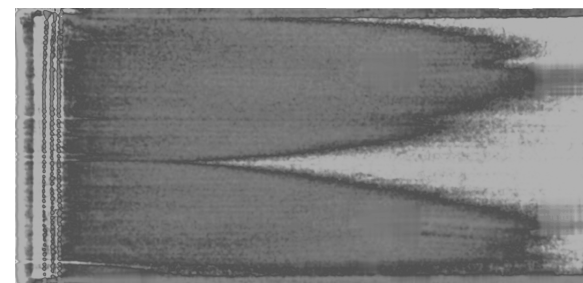

$(c)$ (b)

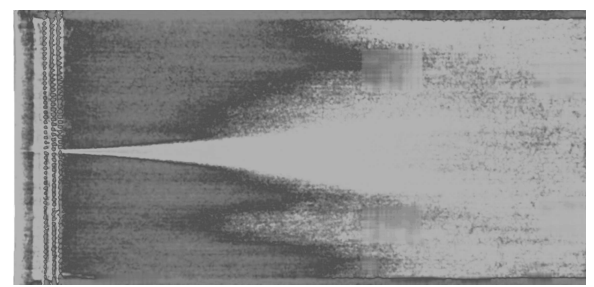

(d)

Figure 3 Heat flux distribution (Stanton number) at $\mathrm{M}=6$, flow - left-to-right: (a) $r=0 \mathrm{~mm}$, with trips; (b) $r=0 \mathrm{~mm}$, without trips; (c) $r=0.75 \mathrm{~mm}$, without trips; and $(d) r=2 \mathrm{~mm}$, without trips. (Refer color plate, p. I.)

tion begins at distance $X_{t} \approx 20 \mathrm{~mm}\left(\operatorname{Re}_{t} \approx 1.1 \cdot 10^{6}\right)$ and ends at $X_{T}=36 \mathrm{~mm}$ $\left(\operatorname{Re}_{T}=2 \cdot 10^{6}\right)$. In section $Z=50 \mathrm{~mm}$, the transition zone is shifted backward: $X_{t}=90 \mathrm{~mm}\left(\operatorname{Re}_{t}=4.9 \cdot 10^{6}\right)$ and $X_{T}=176 \mathrm{~mm}\left(\operatorname{Re}_{T}=9.7 \cdot 10^{6}\right)$.

On the plate with trips, the Stanton numbers are close to the values computed for turbulent boundary layer even at distance $X=50 \mathrm{~mm}$ from the leading edge ( $25 \mathrm{~mm}$ from generators); just some small instability of about $10 \%$ amplitude takes place. It means that the applied trips are quite efficient for $\mathrm{M}=6$.

At Mach number $\mathrm{M}=5\left(\operatorname{Re}_{\infty L}=21.8 \cdot 10^{6}\right)$, the boundary layer transition in section $Z=50 \mathrm{~mm}$ begins at distance $X_{t}=49 \mathrm{~mm}\left(\operatorname{Re}_{t}=3.3 \cdot 10^{6}\right)$ and ends at $X_{T}=95 \mathrm{~mm}\left(\operatorname{Re}_{T}=6.5 \cdot 10^{6}\right)$ (Fig. 5). These data can be compared with results of boundary layer transition investigations made in many wind tunnels of USA on sharp cones [5]. According to these results, the boundary layer transition at $\mathrm{M}=6$ begins at $\operatorname{Re}_{t}=2.9 \cdot 10^{6}$ and at $\mathrm{M}=5$ - at $\operatorname{Re}_{t}=2.7 \cdot 10^{6}$. It can be considered as indirect indication that disturbances level in UT-1 wind tunnel is of the middle value in the sections located apart of the wind tunnel axis. But appropriate Reynolds numbers for flight conditions and for quiet wind tunnels are essentially higher: at $\mathrm{M}=5-6-\mathrm{Re}_{t} \approx 7 \cdot 10^{6}$.

In the central part of the plate at $\mathrm{M}=5$, the boundary layer transition begins near the leading edge, both without and with trips. Trips just decrease transition 


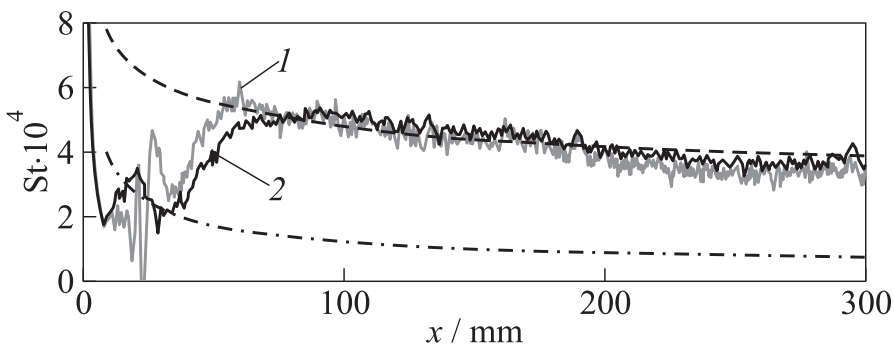

(a)

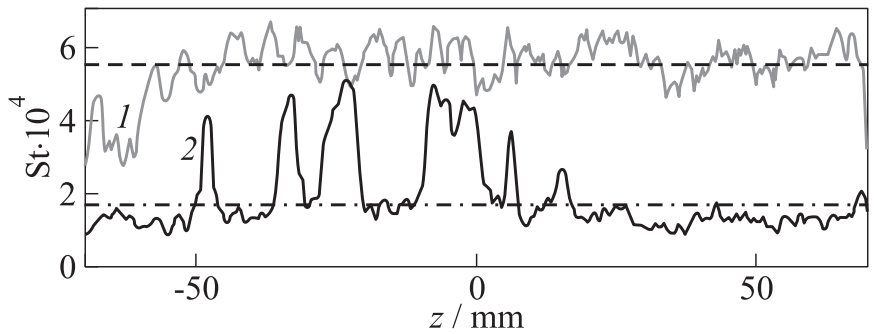

(b)

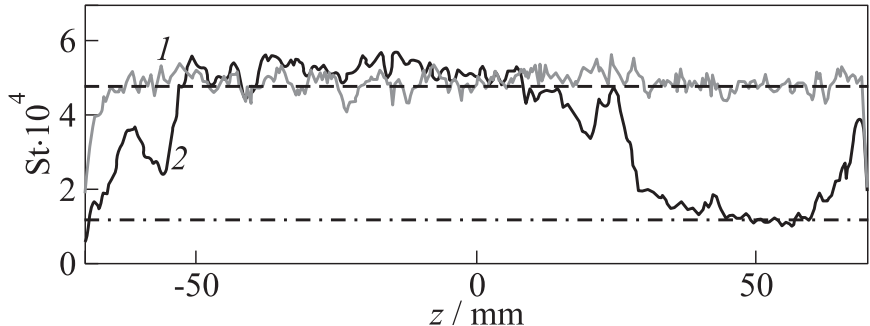

(c)

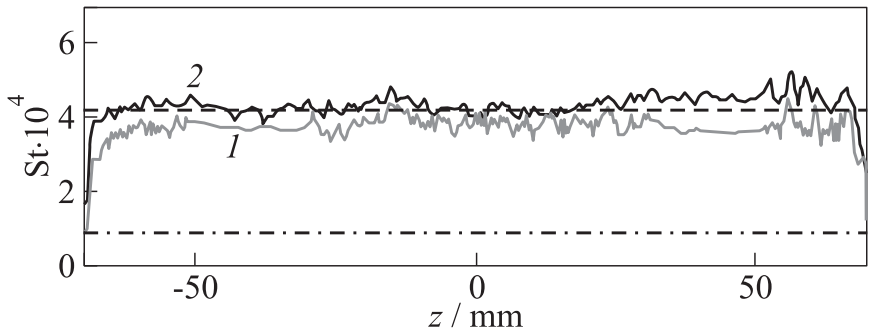

$(d)$

Figure 4 Longitudinal $(a)$ and lateral sections at $\mathrm{M}=6, \operatorname{Re}_{\infty L}=17.5 \cdot 10^{6}$, and $r=0 \mathrm{~mm}(1$ - with trips, 2 - without, dash-dot line - calculated laminar boundary layer, and dash line - turbulent): (b) $X=50 \mathrm{~mm}$; (c) 100; and (d) $X=200 \mathrm{~mm}$ 


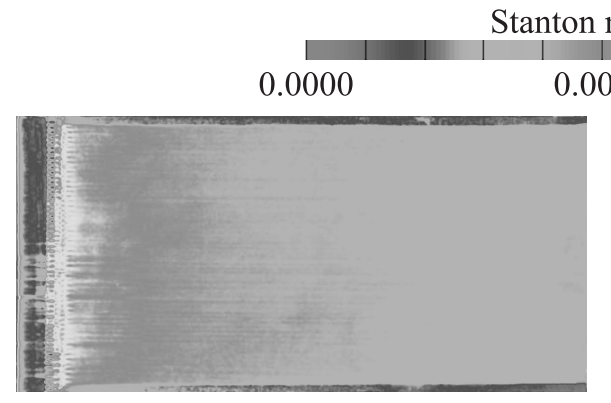

(a) (b)

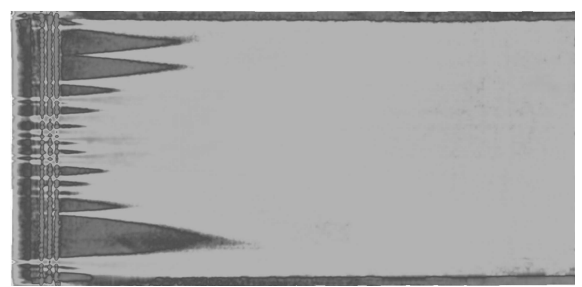

(c)

Figure 5 Heat flux distribution (Stanton number) at $\mathrm{M}=5$, flow - left-to-right: (a) $r=0 \mathrm{~mm}$, with trips; (b) $r=0 \mathrm{~mm}$, without trips; and (c) $r=0.75 \mathrm{~mm}$, without trips. (Refer color plate, p. II.)

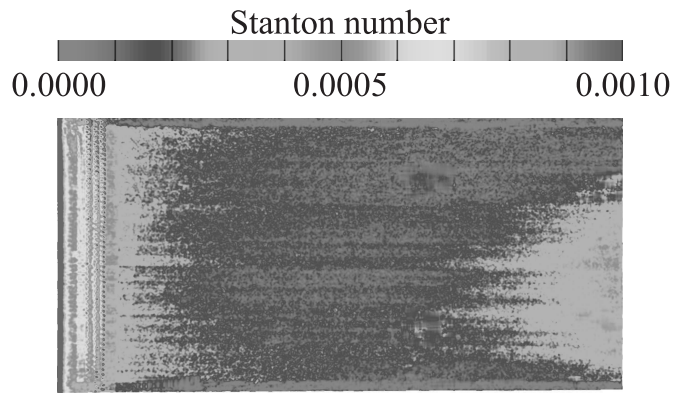

Figure 6 Heat flux distribution (Stanton number) at $\mathrm{M}=8, r=0 \mathrm{~mm}$, with trips, flow left-to-right. (Refer color plate, p. II.) zone: without generators, the transition ends at $X_{T}$ $=50 \mathrm{~mm}$, while with generators - at $X_{T}=25 \mathrm{~mm}$. Apart the center of the plate, the transition also begins immediately after generators and ends at $X_{T}$ $=25 \mathrm{~mm}$.

At higher Mach number $\mathrm{M}=8$, the applied generators do not induce boundary layer transition (Fig. 6). Generator height of $0.2 \mathrm{~mm}$ in this case is ineffective for boundary layer of $0.7 \mathrm{~mm}$ thickness (at the distance of generators installation $X=20 \mathrm{~mm}$ ), but should be $0.6 \mathrm{~mm}$ at least (according to $[4]$ ).

Figures $3 b$ to $3 d$ present an influence of leading edge bluntness on boundary layer transition. Bluntness of 0.75 and $2 \mathrm{~mm}$ radii $(r)$ eliminates all turbulent 


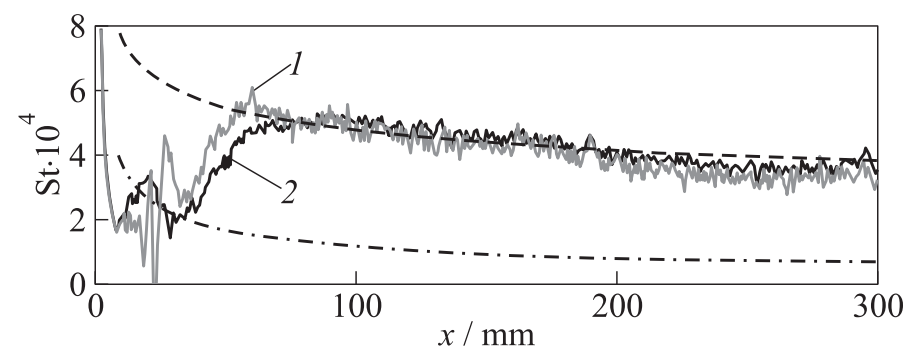

(a)

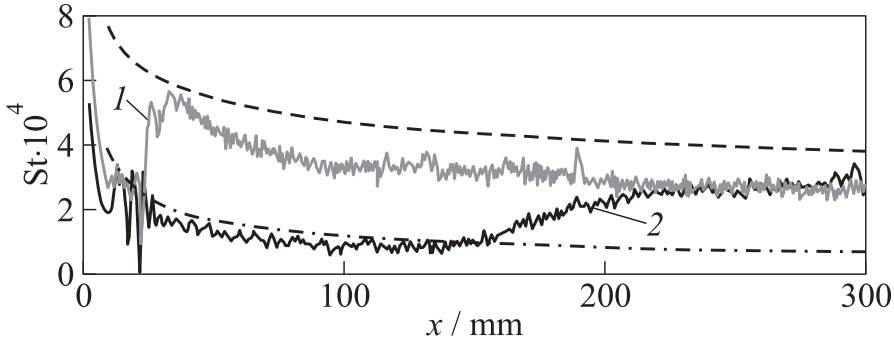

(b)

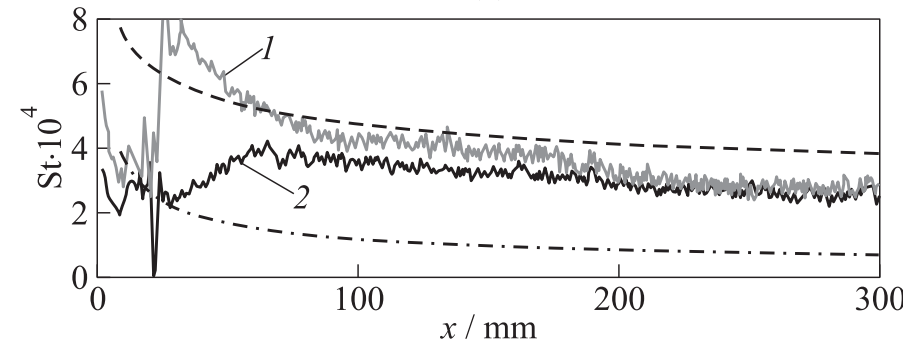

(c)

Figure 7 Longitudinal sections $(Z=0 \mathrm{~mm})$ at $\mathrm{M}=6, \operatorname{Re}_{\infty L}=17.5 \cdot 10^{6}$ at $r=0(a), 0.75(b)$, and $2 \mathrm{~mm}(c)$ (1 - with trips; 2 - without; dash-dot line laminar boundary layer; and dash line - turbulent)

wedges except the wedge in the plate center. It means that disturbances are much higher in the axial part of the flow than in the other parts.

Figure 7 presents central longitudinal sections $(Z=0 \mathrm{~mm})$ of Stanton number at bluntness radii $0,0.75$, and $2 \mathrm{~mm}$. Small leading edge bluntness moves the boundary layer transition backwards, but an increase of bluntness radius leads to some shift of transition forward. Reverse of boundary layer transition at the cone blunting is known $[6,7]$. Backward shift of boundary layer transition at the bluntness increase can be explained easily: it is caused by the decrease of Reynolds number calculated with entropy layer characteristics and by entropy 
layer elongation (before its absorption by boundary layer) at the increase of bluntness radius $r$. But it is more complicated to explain reversive shift of boundary layer transition forward at further increase of bluntness radius. Some authors explain this effect by instability of laminar boundary layer on the bodies of small bluntness or by instability of entropy layer. This problem should be investigated more carefully.

\section{CONCLUDING REMARKS}

Temperature Sensitive Paint method is a very efficient technique for investigations of boundary layer transition in short-duration wind tunnels. It is planed to apply TSP method for transition investigations in adiabatic-compression wind tunnel AT-303 (Khristianovich Institute of Theoretical and Applied Mechanics SB RAS).

\section{ACKNOWLEDGMENTS}

Investigations were performed under financial support of International Science and Technology Center, ISTC Project \# 3872.

\section{REFERENCES}

1. Mosharov, V., A. Orlov, and V. Radchenko. 2003. Temperature sensitive paint (TSP) for heat transfer measurement in short duration wind tunnels. 20th ICIASF. Goettingen, Germany.

2. Borovoy, V., V. Mosharov, V. Radchenko, and A. Noev. 2007. Three-dimensional interference of oblique shock with boundary and high-entropy layers, and techniques intended for investigation of such flows. WEHSFF 2007. Moscow.

3. Potter, J. L., and J. D. Whitfield. 1962. Effects of nose bluntness and roughness on boundary-layer transition in supersonic flows. J. Fluid Mech. 12(Pt. 4):501-35.

4. Morrisette, E. L. 1976. Roughness-induced transition criteria for Space Shuttle-type vehicles. J. Spacecraft Rockets 13(2):118-20.

5. Beckwith, I. E. 1975. Development of a high Reynolds number quiet tunnel for transition research. AIAA J. 13(3):300-6.

6. Stetson, K. F. 1983. Nose tip bluntness effects on cone frustum boundary layer transition in hypersonic flow. AIAA Paper No. 83-1763.

7. Schneider, S. P. 2004. Hypersonic laminar-turbulent transition on circular cones and scramjet forebodies. Prog. Aerospace Sci. 40(1-2):1-50. 